\title{
Erratum to: Dynamical diagram and scaling in polymer driven translocation
}

\author{
Takuya Saito ${ }^{1, a}$ and Takahiro Sakaue ${ }^{1,2, b}$ \\ 1 Department of Physics, Kyushu University 33, Fukuoka 812-8581, Japan \\ 2 PRESTO, Japan Science and Technology Agency (JST), 4-1-8 Honcho Kawaguchi, Saitama 332-0012, Japan
}

Original article: Eur. Phys. J. E (2011) 34: 135, DOI: 10.1140/epje/i2011-11135-3

Received 18 May 2012 and Received in final form 6 August 2012

Published online: 29 November 2012 - (C) EDP Sciences / Società Italiana di Fisica / Springer-Verlag 2012

In this erratum, we make a correction of steady-state ansatz $V(t) \equiv v(t, x=-R)$ used in ref. [1]. The same ansatz was also adopted in our earlier publications $[2,3]$, where the basic framework for the driven translocation was proposed. However, as discussed in [4], one should instead set the steady-state velocity $V(t) \equiv v(t, x=0)$ for the steady-state approximation to be self-consistent.

With this modification only in mind, the following procedure remains almost intact. To be more precise, we here provide a rough sketch of the analysis (see ref. [5] for detailed discussion). From the definition of the current at the pore

$$
\frac{\mathrm{d} M}{\mathrm{~d} t}=\sigma(x=0) V(t)
$$

one can construct the dynamical scaling of the tension front propagation in the driven translocation process in the following way. Essential ingredients are: i) the dynamical equations of state [6] for the moving domain

$$
\begin{aligned}
V(t) R(t) & \simeq f^{p_{z}}, \\
N(t)-M(t) & \simeq R(t) f^{-p_{\nu}},
\end{aligned}
$$

where $\left(p_{z}, p_{\nu}\right)=(z-2,(1-\nu) / \nu)$ in the trumpet regime $k_{B} T / R_{0}<f<k_{B} T / a$ and $\left(p_{z}, p_{\nu}\right)=(1,0)$ for stronger force, and ii) the average initial configuration

$$
N(t)^{\nu} \simeq R(t)
$$

From eqs. (1)-(4), one can obtain the differential equation for $R(t)$, which leads to the asymptotic scaling for the tension propagation time

$$
\tau_{\mathrm{p}} \simeq N_{0}^{1+\nu} f^{-\left(p_{z}-p_{\nu}\right)}= \begin{cases}N_{0}^{1+\nu} f^{1+(1 / \nu)-z}, & \left(N_{0}^{-\nu} \lesssim f \lesssim 1\right) \\ N_{0}^{1+\nu} f^{-1}, & (1 \lesssim f)\end{cases}
$$

As the tension propagation stage dominates the post-propagation stage in the scaling limit, this is identified with the translocation time scaling $\tau \simeq \tau_{\mathrm{p}}$.

The modified ansatz is compatible with the iso-flux model proposed by Rowghanian and Grosberg [7], which is discussed in ref. [5] in detail. A recent work by Dubbeldam et al. has also adopted the same ansatz, thus, ended up with essentially the same result [8]. The asymptotic scaling $\tau \sim N_{0}^{1+\nu}$ has recently been verified by Ikonen et al., using the Brownian dynamics tension propagation model [9].

\footnotetext{
a Present address: Fukui Institute for Fundamental Chemistry, Kyoto University, Kyoto 606-8103, Japan. e-mail: saito@fukui.kyoto-u.ac.jp

b e-mail: sakaue@phys.kyushu-u.ac.jp
} 


\section{References}

1. T. Saito, T. Sakaue, Eur. Phys. J. E 34, 135 (2011).

2. T. Sakaue, Phys. Rev. E 76, 021803 (2007).

3. T. Sakaue, Phys. Rev. E 81, 041808 (2010).

4. T. Saito, T. Sakaue, Phys. Rev. E 85, 061803 (2012).

5. T. Saito, T. Sakaue, arXiv:1205.3861 [cond-mat.soft].

6. T. Sakaue, T. Saito, T. Wada, Phys. Rev. E 86, 011804 (2012).

7. P. Rowghanian, A.Y. Grosberg, J. Phys. Chem. B 115, 14127 (2011).

8. J.L.A. Dubbeldam, V.G. Rostiashvili, A. Milchev, T.A. Vilgis, Phys. Rev. E 85, 041801 (2012).

9. T. Ikonen, A. Bhattacharya, T. Ala-Nissila, W. Sung, Phys. Rev. E 85, 051803 (2012). 